\title{
DATAÇÃO KJAr DE ARGILAS ASSOCIADAS ÀS MINERALIZAÇÕES E AOS PROCESSOS DIAGENÉTICOS, EM RELAÇÃO COM A HISTÓRIA DA ABERTURA DO OCEANO ATLÂNTICO SUL
}

\author{
ROSA P. SANTOS* \& MICHEL G. BONHOMME*
}

\begin{abstract}
K/AR DATING OF CLAYS ASSOCIATED WITH MINERALIZATIONS, AND DIAGENETIC PROCESSES, IN RELATION WITH THE SOUTH ATLANTIC OCEAN BREAKUP HISTORY. K/Ar dating of clays associated with some vein ore-deposits and with diagenetic alterations were performed on samples collected from various locations along the Atlantic coast of South America and in the Parana Basia After mineralogical and geochemical control, especially concerning the TiO content, the calculated ages show the following results. 1. The ages of clays associated with fluorite mineralizations of the Santa Catarina Mine (SC) and uranium deposits at Poços de Caldas (MG) are 87 and 67,5 Ma, respectively. 2. The ages of clays from the 2TB 1SP hole in the Parana Basin define an older high temperature diagenetic episode dated between 140 and $130 \mathrm{Ma}$ and a subsequent lower temperature event dated between 120 and $100 \mathrm{Ma}$. 3. K/Ar determinations of celadonites and apophylites of diagenetic alterations of the Serra Geral flood basalts put this event between 120 and $100 \mathrm{Ma}$. 4. All these results can be correlated with the geodynamic and magmatic evolution of South America during the breakup of the South Atlantic Ocean. 5. The relationship in space and time between diagenesis and vein-type mineralizations on one hand and tensional tectonics on the other hand was already defined and accepted by various authors along the North Atlantic. Comparison between the new data of the present work with those used to define the model for North Atlantic lead to the conclusion that the same mechanisms have been operating during South Atlantic breakup.
\end{abstract}

Keywords: Clays, mineralizations, diageneses, dating, K/Ar, tensional tectonics, continental breakup.

\begin{abstract}
RESUMO Datações K/Ar das argilas ligadas a certas mineralizações filonianas e às transformações diagenéticas foram efetuadas sobre as amostras provenientes de diversas localidades ao longo da costa atlântica da América do Sul e da Bacia do Paraná. Após controle tnineralógico e geoquímico, particularmente sobre o teor em titânio das argilas, as idades obtidas mostram os seguintes resultados: 1. As datações em argilas ligadas as mineralizações de fluorita da Mina Santa Catarina (SC) e de urânio em Poços de Caldas (MG) forneceram idades de 87 e 67,5 Ma, respectivamente. 2. As datações em argilas de diagênese da Bacia do Paraná permitiram precisar a existência de um episódio diagenético de alta temperatura entre 140 e $130 \mathrm{Ma}$ e outro de baixa temperatura entre 120 e $100 \mathrm{Ma}$. 3. As datações em celadonitas e apofllitas de alteração diagenética nos basaltos da Formação Serra Geral colocam este evento entre 120 e 110 Ma. 4. Todos os dados acima podem ser situados direta ou indiretamente dentro do contexto da abertura do Oceano Atlântico Sul e dos fenômenos magmáticos decorrentes. 5. A ligação no espaço e no tempo, entre diagênese e mineralizações filonianas, de um lado, e tectônica extensional, do outro, já foi observada por diversos autores ao longo do Atlântico Norte. Estes dados apresentam mecanismos idênticos ocorridos durante a abertura do Atlântico Sul.
\end{abstract}

Palavras-chave: Argilas, mineralizações, diagênese, datação, K/Ar, tectônica extensional, abertura continental.

INTRODUÇÃO O conceito ligando as mineralizações filonianas situadas na interface embasamento/cobertura sedimentar com a tectônica extensional foi sugerido, baseado em dados radiocronológicos, por Bonhomme $(1982,1987)$ e Bonhomme et al. $(1983,1987)$. Estudos do mesmo tipo, efetuados por Halliday et al. (1984) e Respaut et al. (1990) confirmaram também o modelo. Por outro lado, utilizando apenas dados tectônicos. Touray e Bellon (1976), Jebrak (1984) e Bastos et al. (1991) chegaram às mesmas conclusões. Bonhomme (1987) mostrou também a relação no espaço e no tempo entre os veios filonianos mineralizados e os episódios de diagênese que foram datados, por exemplo, na Bacia de Paris (Mossmann 1987,1991)e no Mar do Norte (Liewig et al 1987). O objetivo deste artigo foi o de mostrar se este conceito se aplicaria também à abertura do Atlântico Sul, depois de Ter sido definido na Europa Ocidental em relação ao Atlântico Norte e o Oceano Ligure. Para a realização deste trabalho, foram coletadas amostras nos seguintes domínios: argilas associadas aos filões de fluorita e em algumas jazidas de urânio; argilas diagenéticas da Bacia do Paraná; apofllitas e celadonitas sedundárias cristalizadas dentrodosbasaltosdaBacia do Paraná.

\section{AMOSTRAGEM, MÉTODOS E MATERIAIS UTILIZADOS As amostras associadas às mineralizações foram}

coletadas nas proximidades imediatas dos minérios. As argilas foram coletadas ora ao longo dos filões, ora no interior do minério, dentro das fissuras ou das cavidades, ora dentro das fraturas da encaixante imediatamente próximas ao minério, nos casos menos favoráveis. Um cuidado todo particular foi tomado para evitar, ao máximo, as argilas de superficie, para limitar os efeitos da intemperização, e as argilas de cor ocre, visto que o oxido de ferro é, por experiência, um indicador freqüente de circulação vindo da superfície. Esta precaução revela-se indispensável se observar-se que a quase totalidade da área de estudo situa-se em regime de clima tropical úmido. O material analisado é freqüentemente uma pasta argilosa ainda úmida. Basta colocar esta pasta argilosa no aparelho de ultra-som para se obter a suspensão e proceder a separação das argilas.

As argilas das amostras de sondagem foram selecionadas ao microscópio eletrônico de varredura.

As celadonitas foram extraídas de basaltos, dos vacúolos de ágata, quartzo ou ametista. As apofllitas, provenientes de amostras de coleção, foram moídas primeiramente e as frações peneiradas entre $315-400 \mathrm{~mm}$ foram tomadas para estudos.

LOCALIZAÇÃO DAS AMOSTRAS As amostras dos veios mineralizados são provenientes das seguintes localidades: - Minas dê fluorita Santa Catarina e Nossa Senhora do 
Carmo, Estado de Santa Catarina (Bastos Neto et al. 1991), sobre um filão orientado N20E.

- Mina de urânio de Poços de Caldas, Estado de Minas Gerais.

A amostra 87 MB 120A provém de um filão de fluorita, com orientação EW ao sul de Serra Grande, Patagônia, Argentina.

As argilas diagenéticas foram escolhidas no poço profundo 2 TB - 1 SP a partir dos testemunhos de sondagens dentro da Formação Furnas, de idade siluriana (Zalán et al 1986). A profundidade da coleta é de 4.500 a $4.910 \mathrm{~m}$ dentro de uma das zonas mais profundas da'Bacia do Paraná.

As celadonitas foram extraídas de amígdalas de basaltos microporfirí ticos da região de Planalto, Estado do Rio Grande do Sul, Brasil. As apofilitas analisadas são fragmentos de amostras de coleção, coletadas dentro dos vacúolos de basaltos. Estes dois tipos de amostras testemunham os mecanismos de alteração interna dos basaltos da Formação Serra Geral, de idade jurássica superior a cretácica inferior, na Bacia do Paraná, Brasil (Rocha Campos et al 1989).

TÉCNICAS ANALÍTICAS As argilas provenientes de filões mineralizados foram todas tratadas diretamente no aparelho de ultra-som, enquanto as amostras de sondagem foram moídas moderadamente antes do mesmo tratamento. As celadonitas foram retiradas da superfície das amígdalas com uma espátula ou com uma broca rotativa. A mineralogia das frações finas foi definida segundo regras propostas por Moore \& Reynolds (1989), particularmente a classificação dos interestratifícados I/S. Estes são definidos como Ro à R3, conforme os teores crescentes em illita e, conseqüentemente, às qualidades crescentes da ordem cristalina do mineral. A dosagem do potássio foi obtida por fluorescência de raios X (Govindaraju 1987), por ICP para as amostras pobres em potássio ou por sonda iônica para as celadonitas (média de 8 a 24 pontos de análise). A dosagem do argônio radiogênico foi efetuada por diluição isotópica empregandose um espectrômetro de massa VG série 600. Para o cálculo de idade foram utilizadas as constantes recomendadas por Steiger \& Jàger (1977), enquanto para o cálculo de erro foi adotada a fórmula estabelecida por Mahood \& Drake (1982). Os erros foram de mais ou menos $3 \%(1 \sigma)$ para as amostras contendo mais de $50 \%$ de argônio radiogênico. A tabela 1 contém os valores obtidos no Instituto Dolomieu em Grenoble, para cada padrão internacional durante a elaboração deste trabalho comparados aos valores publicados na literatura.

Tabela 1 - Teores médios em ${ }^{40}$ Ar rad. de minerais padrões internacionais

Table $1-{ }^{40} \mathrm{Ar}$ rad. mean contents of the international standard minerals

\begin{tabular}{|c|c|c|c|c|}
\hline 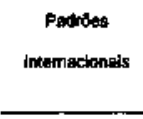 & 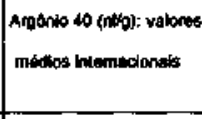 & $\begin{array}{c}\omega_{\text {Ar rad. }} \\
(\omega)\end{array}$ & 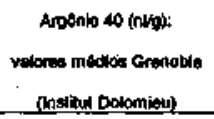 & $\begin{array}{l}\text { Nùmono de } \\
\text { mentremen }\end{array}$ \\
\hline Slouranite $\mathrm{GL}$-O & 겨농 & $\infty .0$ & $25,125 \pm 0,033(10)$ & 13 \\
\hline Bestla $-A D-a_{1}$ & 7,70 & 0 & $7,0001 \pm 0,235,(10)$ & 11 \\
\hline Englien Ipsero & 43,18 & po.gs & $43,70 \pm 0.95,110)$ & 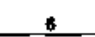 \\
\hline Evellta 48 & 5.39 & -90 & $3.632 \pm 0.181110$ & 1 \\
\hline
\end{tabular}

RESULTADOS OBTIDOS A tabela 2 apresenta os dados mineralógicos e a tabela 3 contém os dados analíticos $\mathrm{K} / \mathrm{Ar}$.

Alguns destes dados aparecem publicados em Dos Santos \& Bonhomme (1991), os quais foram modificados após verificação dos teores em potássio.
No que se refere às amostras provenientes da Jazida de Poços de Caldas, os dados isotópicos ${ }^{40} \mathrm{~K}{ }^{\beta 6} \mathrm{Ar} e^{40} \mathrm{Ar} /{ }^{36} \mathrm{Ar}$ alinham-se no diagrama isocrônico K/Ar, coforme mostra a figura 1. Os parâmetros da reta obtida fornecem as seguintes indicações: $t=67,5 \pm 2,7 \mathrm{Ma}( \pm 1 \sigma), \mathrm{MSWD}=0,72 . \mathrm{O}$ valor inferior a 1 para MSWD confirma que a dispersão dos pontos é somente de origem analítica (Williamson 1968).

$\mathrm{O}$ mesmo tipo de ajustamento pode ser proposto para as celadonitas, que são todas provenientes do mesmo nível de basalto (Fig. 2). A reta englobando quatro das cinco celadonitas analisadas fornece: $\mathrm{t}=109,3 \pm 2,9 \mathrm{Ma}( \pm 1 \sigma)$,

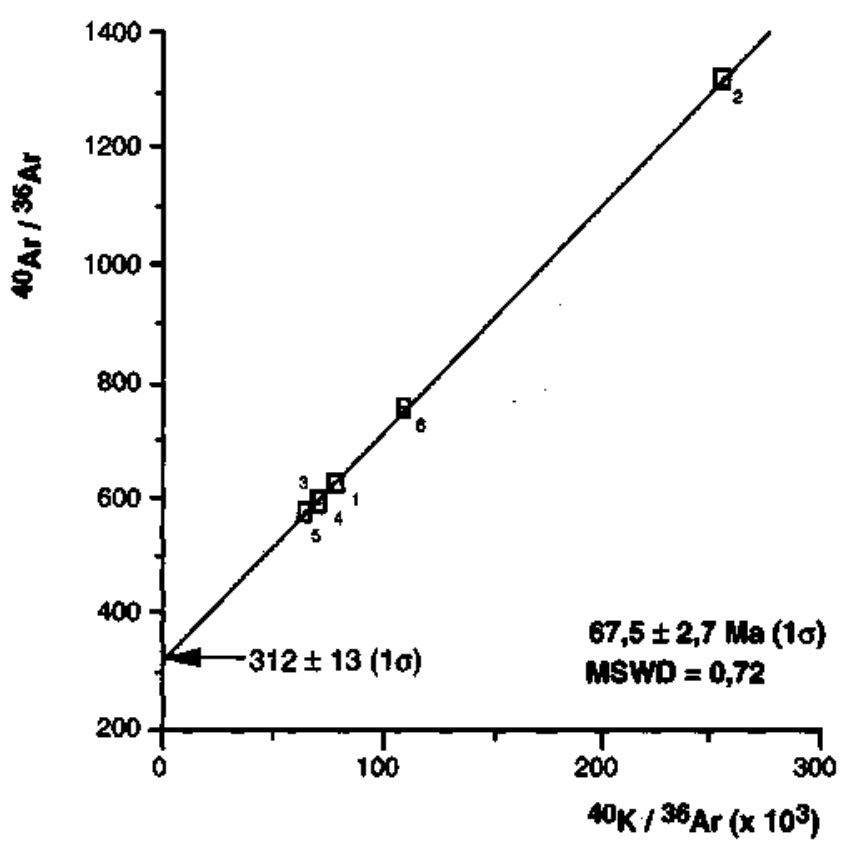

Figura 1 - Diagrama isotópico K/Ar em frações $<2$ jum de argilas associadas a urânio em Poços de Caldas (MG-Brasil) Figure $1-\mathrm{K} / \mathrm{Ar}$ isotopic diagramme of fractions $<2 \mu \mathrm{m}$ of clays associated with uranium deposit in Poços de Caldas (MG - Brasil)

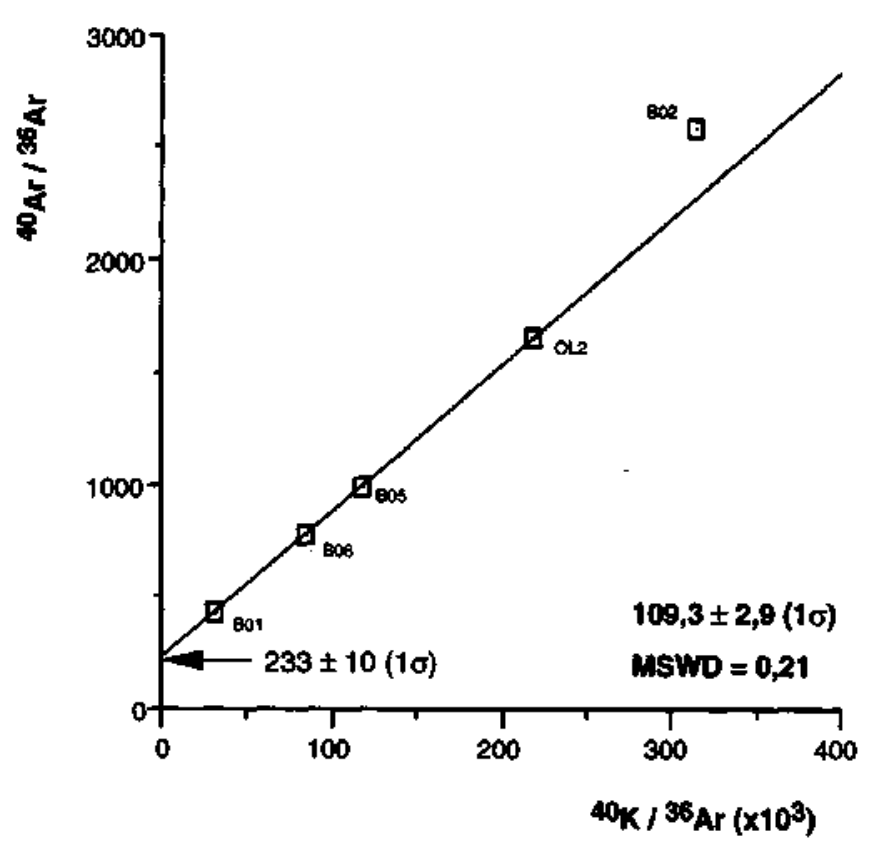

Figura 2 - Diagrama isotópico K/Ar em Celadonitas da região de Planalto ( $R S$ - Brasil)

Figure $2-K / A r$ isotopic diagramme of celadonites in the Planalto region (RS -Brasil) 
Tabela 2 - Dados mineralógicos das frações argilosas $<2 \mu \mathrm{m}$

Table 2 - Mineralogical data of the clay fractions $<2 \mu \mathrm{m}$

\begin{tabular}{|c|c|c|c|c|c|c|}
\hline $\begin{array}{l}\text { Número da } \\
\text { amostra }\end{array}$ & $\begin{array}{c}\text { llita } \\
\%\end{array}$ & $\begin{array}{c}\text { Caol. } \\
\%\end{array}$ & $\begin{array}{c}\text { Clor } \\
\%\end{array}$ & $\begin{array}{c}S \mathrm{Sm}+\mathrm{I} / \mathrm{S} \\
\%\end{array}$ & $\begin{array}{c}\text { Talco } \\
\%\end{array}$ & Acessórios \\
\hline 88 RPSG12A & tr. & 50 & & $50(\mathrm{Sm})$ & & \\
\hline 88 RPSG16 A & & 50 & & $45(\mathrm{Sm}) 5(\mathrm{Ro})$ & & Goetita* \\
\hline 88 RPSG17 A & & 30 & & $70(\mathrm{Sm}) \operatorname{tr}(\mathrm{RO})$ & & \\
\hline $87 \mathrm{MB} \overline{46 \mathrm{~A}}$ & $\begin{array}{c}\text { 10Tabela } 2 \text { : Dados } \\
\text { mineralogicos das fraçбos } \\
\text { argilosas }<2 \mu \mathrm{m} \\
0\end{array}$ & & & & & \\
\hline $87 \mathrm{MB} 47 \mathrm{~A}$ & 65 & & 5 & $30(30 \%$ ill,RO) & & $Q^{*}$ \\
\hline $87 \mathrm{MB} 120 \mathrm{~A}$ & & & & $\begin{array}{c}(60 \% \text { ill, R1) } 40 \\
(<10 \% \text { ill,RO)60 } \\
\end{array}$ & & \\
\hline $87 \mathrm{MB} 01 \mathrm{~A}$ & & 100 & & & & KF \\
\hline $87 \mathrm{MB} 04 \mathrm{~A}$ & 30 (crist $=0,30^{\circ} ; 2 / 1=0,43$ ) & 70 & & & & KF** \\
\hline $87 \mathrm{MB} 05 \mathrm{~A}$ & 10 & 90 & & & & KF *n*k \\
\hline $87 \mathrm{MB} 06 \mathrm{~A}$ & $20\left(\right.$ crist. $\left.=0,20^{\circ} ; 2 / 1=0,39\right)$ & 80 & & & & KF *\# \\
\hline $87 \mathrm{MB} 12 \mathrm{~A}$ & 20 & 80 & & & & KF ** \\
\hline $87 \mathrm{MB13 \textrm {A }}$ & 25 (crist. $=0,20^{\circ} ; 2 / 1=0,35$ ) & 75 & & & & KF ** \\
\hline \multicolumn{7}{|l|}{2 TB 1 SP } \\
\hline 4494,2 & $\begin{array}{c}100\left\langle\text { crist }=0,25^{\circ} ; 2 / 1=0,39\right) 2 \mathrm{M} \\
\text { pura }\end{array}$ & & $<5$ & & & \\
\hline 4494,8 & $\begin{array}{c}\left.100 \text { (crist }=0,26^{\circ} ; 2 / 1=0,39\right) 2 M \\
\text { pura }\end{array}$ & & $<5$ & & & \\
\hline $4694 ; 9 \mathrm{~A}(1)$ & ill/par $50 / 50,702 / 1=0,33)$ & 30 & & & & $Q^{*}$ \\
\hline $4695,9 \mathrm{~B}(1)$ & ill/par 50/50,70 $2 / 1=0,33$ & 30 & & & & $\mathrm{Q}^{*}$ \\
\hline $4910,0 \mathrm{~A}(2)$ & 85 (Crist $=0,28^{\circ} ; 2 / 1=0,37$ & & 15 & & & $\mathrm{Q}^{* * *}, \mathrm{KF}^{\star}, \mathrm{PI}^{*}$ \\
\hline $4910,0 \mathrm{~B}(2)$ & 85 (Crist $\left.=0,36^{\circ} ; 2 / 1=0,35\right)$ & & 15 & & & $\mathrm{Q}^{\star \star \star \star}, \mathrm{KF}^{\star \star \star},\left.\mathrm{P}\right|^{\star \star \star}$ \\
\hline
\end{tabular}

(1) Amostra B melhor cristalizada que A; ill/par = interestratificado illita-paragonita; mineral de 7A do tipo dickita

(2) Amostra A melhor cristalizada que $B$

* presente

** abundante

*** muito abundante

$\left({ }^{40} \mathrm{Ar} /{ }^{36} \mathrm{Ar}\right) \mathrm{O}=233 \pm 10( \pm 1 \sigma)$ eMSWD $=0,21$. A intersecção $\left({ }^{40} \mathrm{Ar} /{ }^{36} \mathrm{Ar}\right) \mathrm{o}$ é significativamente inferior a 295,5 . Este valor é interpretado como impossível e que se trataria de uma reta de mistura, portanto sem significado. Uma outra possível interpretação seria a de admitir que. a partir de uma isócrona com uma intersecção normal, teria havido perdas de argônio radiogênico ou ganhos de potássio. Esta última possibilidade seria a menos provável, porque as quatro amostras sobre a reta são as mais pobres em potássio.

DISCUSSÃO DOS RESULTADOS Relação entre os dados mineralógicos, geoquímicos e isotópicos. As amostras das Minas Nossa Senhora do Carmo e Santa Catarina serão abordadas juntamente, porque elas são provenientes do mesmo filão (Bastos Neto 1990).

As amostras da Mina Nossa Senhora do Carmo contêm uma mistura de caolinita e de esmectita com um pouco de interestratificado $1 / \mathrm{S}$ do tipo Ro. Os teores em óxidos de titânio $\left(\mathrm{TiO}_{2}\right)$ dessas amostras variam entre 0,35 e $0,78 \%$. Ao contrário, as amostras da Mina Santa Catarina contém 65 ou $100 \%$ de illita, 0 a $5 \%$ de clorita e 0 ou $30 \%$ de interestratificado 1/S do tipo Ro. Os teores em oxido de titânio destas amostras são de mais ou menos $0,10 \%$. As idades das amostras da Mina Nossa Senhora do Carmo variam entre
$160 \mathrm{Ma}$ e $239 \mathrm{Ma}$, enquanto as duas amostras datadas da Mina Santa Catarina forneceram idades de $86 \mathrm{Ma}$ e $87 \mathrm{Ma}$. A natureza mineralógica e os teores em titânio das amostras da Mina Nossa Senhora do Carmo são típicas de material ígneo, com intemperização atual (Bonhomme 1987). Inversamente, as amostras da Mina Santa Catarina, illíticas, isentas de caolinita e pobres em oxido de titânio, são características de argilas hidrotermais. A idade de cerca de $87 \mathrm{Ma}$, ou seja, Coniaciano, deve então ser considerada como idade do evento mineralizador (Haq \& Van Eysinga 1987). Contudo, não é possível atribuir esta idade a uma das quatro fases tectônicas observadas por Bastos Neto et al. (1991). Porém, esta datação confirma bem a idade cretácica sugerida por esses autores. As idades aparentes obtidas na Mina Nossa Senhora do Carmo são ligadas à intemperização mais ou menos forte de amostras da rocha encaixante. Quando a proporção de interestratificados Ro aumenta, a idade diminui. Conseqüentemente, esses interestratificados podem ser considerados como testemunhos da transformação hidrotermal. Mas, como esta transformação não se realizou completamente na localidade da Mina Nossa Senhora do Carmo, as "idades" calculadas não têm nenhum significado geológico.

As argilas de Poços de Caldas provém de duas sondagens exploradas na jazida de urânio. As amostras são fragmentos de testemunhos coletados sob o limite de variação das napas 
Tabela 3 - Dados analíticos potássio-argônio

Table 3 - Potassium-argon analytical data

\begin{tabular}{|c|c|c|c|c|c|c|}
\hline Localizaçăo & $\begin{array}{l}\text { Número da } \\
\text { amostra }\end{array}$ & $\begin{array}{l}\mathrm{K}_{2} \mathrm{O} \\
(\%)\end{array}$ & $\begin{array}{c}40 \mathrm{Ar} \cdot \mathrm{rad} \\
(\%)\end{array}$ & $\begin{array}{c}\text { 40Ar rad } \\
\text { (nl/g) }\end{array}$ & $\begin{array}{c}\mathrm{TiO2} \\
(\%)\end{array}$ & $\begin{array}{c}t \\
(\operatorname{Ma}+1 \sigma)\end{array}$ \\
\hline \multirow{3}{*}{$\begin{array}{l}\text { Nossa.Senhora } \\
\text { do Camo' (SC) }\end{array}$} & 88 RPSG $12 \mathrm{~A}$ & 1,38 & 87,5 & 11,45 & 0,53 & $239 \pm 7$ \\
\hline & 88 RPSG $16 \mathrm{~A}$ & 1,59 & 76,9 & 8,61 & 0,78 & $160,5 \pm 4,5$ \\
\hline & 88RPSG 17 A & 1,25 & 66,1 & 7,82 & 0,36 & $184,1+6,4$ \\
\hline \multirow{2}{*}{$\begin{array}{c}\text { Santa } \\
\text { Catarina (SC) }\end{array}$} & $87 \mathrm{MB} \mathrm{46} \mathrm{A}$ & 8,53 & 75,2 & 24,6 & 0,13 & $87,3+2,0$ \\
\hline & $87 \mathrm{MB} 47 \mathrm{~A}$ & 7,10 & 75,2 & 20,1 & 0,08 & $85,8+2,2$ \\
\hline \multirow{2}{*}{$\begin{array}{c}\text { Serra Grande } \\
\text { (Patagónia, Arg.) }\end{array}$} & $87 \mathrm{MB} 120 \mathrm{~A}$ & 1,55 & 39,4 & 5,76 & n.d. & $117,7+7,7$ \\
\hline & & & & & & \\
\hline \multirow{6}{*}{$\begin{array}{c}\text { Pocos de } \\
\text { Caldas (MG) }\end{array}$} & $87 \mathrm{MB} 01 \mathrm{~A}$ & 5,95 & 52,4 & 13,73 & n.d. & $70,1+1,8$ \\
\hline & $87 \mathrm{MB} 04 \mathrm{~A}$ & 10,83 & 77,6 & 24,12 & n,d. & $67,7 \pm 1,7$ \\
\hline & $87 \mathrm{MB} 05 \mathrm{~A}$ & 8,24 & 50,4 & 19,48 & n.d. & $71,8+1,8$ \\
\hline & $87 \mathrm{MB} 06 \mathrm{~A}$ & 8,46 & 49,8 & 19,19 & n.d. & $69,0+1,8$ \\
\hline & $87 \mathrm{MB} 12 \mathrm{~A}$ & 6,17 & 48,7 & 14,84 & n.d. & $73,0 \pm 2,4$ \\
\hline & $87 \mathrm{MB} 13 \mathrm{~A}$ & 9,67 & 61,1 & 22,87 & n.d. & $71,8 \pm 2,1$ \\
\hline \multirow{5}{*}{$\begin{array}{l}\text { Celadonilas } \\
\text { (Planalto, RS) }\end{array}$} & $\mathrm{BO} 1 \mathrm{~A}$ & 7,20 & 31,5 & 17,87 & 0,025 & $75,3 \pm 2,9$ \\
\hline & $\mathrm{BO} 2 \mathrm{~A}$ & 7,97 & 88,6 & 32,4 & n.d. & $121,7+3,2$ \\
\hline & OL 2A & 7,25 & 82,1 & 24,9 & n.d. & $103,3 \pm 2,9$ \\
\hline & $B O 5 A$ & 7.28 & 70,2 & 24,4 & 0,04 & $101,1 \pm 8,4$ \\
\hline & $\mathrm{BO} 6 \mathrm{~A}$ & 7,52 & 62,4 & 24,5 & 0,02 & $98,4 \pm 12,2$ \\
\hline \multirow{2}{*}{$\begin{array}{c}\text { Apofilitas } \\
\text { (De Coleçăo) }\end{array}$} & EVZO1AZ & 4,17 & 71,7 & 15,19 & & $109,5+3,9$ \\
\hline & EVZO1B Z & 4,10 & 80,2 & 15,85 & & $116,0 \pm 5,7$ \\
\hline \multirow{6}{*}{$\begin{array}{c}\text { Argilas } \\
\text { diagenéticas, } \\
\text { Fm Furnas } \\
\text { Bacia do } \\
\text { Paraná - Poço } \\
2 \text { TB 1SP }\end{array}$} & $4494,2^{*}$ & 8,81 & 90,3 & 40.7 & (1) & $137,7 \pm 3,1$ \\
\hline & $4494,8^{*}$ & 8,83 & 89,7 & 39,9 & (1) & $134,9 \pm 3,0$ \\
\hline & $4694,9^{\circ}$ & 3,36 & 51,9 & 14,46 & (1) & $128,7+3,6$ \\
\hline & $4695,0^{*}$ & 3,33 & 48,7 & 15,03 & (1) & $134,7 \pm 4,0$ \\
\hline & $4910,0^{*}$ & 5,98 & 72,4 & 23,79 & (1) & $119,3+2,8$ \\
\hline & $4911,0^{*}$ & 5,12 & 71,4 & 17,34 & (1) & $102,0+2,7$ \\
\hline
\end{tabular}

n.d.: não determinado; (1) não-detectado por fluorescência de raios X sobre os minerais observados ao M.E.V.; $\left(^{*}\right)$ profundidade em metros

freáticas superficiais, para evitar os efeitos de intemperização. A mineralogia dessas argilas é constituída de caolinita com uma pequena quantidade de illita. Todavia, todas as frações, quando analisadas por RX em pó não-orientado, contém uma grande proporção de feldspato potássico que explica ps teores elevados em potássio. A mistura datada é constituída essencialmente de feldspato potássico. Tomando-se em conta os erros de medida, todas as idades são idênticas, o que justifica a apresentação dos resultados sobre a forma de um diagrama isotópico (Fig. 2). $\mathrm{O}$ valor da razão inicial $\mathrm{C}^{10} \mathrm{Ar} /{ }^{8} \mathrm{Ar}$ ) de $311 \pm 12( \pm 1 \mathrm{o})$ é próximo da razão $\left({ }^{40} \mathrm{Ar} /{ }^{36} \mathrm{Ar}\right)$ do argônio atmosférico atual, ou seja, 295,5. Isso significa que as frações analisadas transformaram-se novamente em sistemas fechados ao mesmo tempo e sem excesso de argônio radiogênico. A idade obtida de $67,5 \mathrm{Ma}$ corresponde ao Maestrichtiano. Esta idade é coerente, porque o Maciço Alcalino de Poços de Caldas faz parte do magmatismo alcalino do Atlântico Sul de idade mesozóica (Bristow 1985), e a alteração que acompanha a mineralização uranífera é necessariamente mais jovem. Como as idades das rochas alcalinas da região variam entre
$63 \mathrm{Ma}$ e $80 \mathrm{Ma}$ (Amaral et al. 1967), a idade de 67,5 Ma para a alteração hidrotermal é certamente plausível.

A argila procedente da Patagônia, Argentina, foi coletada em um filão EW cortando as rochas vulcânicas jurássicas. A idade obtida, $120 \mathrm{Ma}$, corresponde conseqüentemente bem a um episódio mais jovem que a ocorrência destas rochas vulcânicas jurássicas, datadas em cerca de $170 \mathrm{Ma}$.

As argilas diagenéticas, extraídas da Formação Furnas, de idade siluriana, no poço 2 TB - 1 SP perfurado na Bacia do Paraná, forneceram idades convencionais que variam entre 138 a $102 \mathrm{Ma}$. Estas idades estão situadas em um período de tempo mais velho que as idades das argilas ligadas aos veios filonianos, com apenas uma exceção (102 Ma).

$\mathrm{O}$ exame mineralógico dessas argilas revela que as duas amostras menos profundas $(\sim 4.494 \mathrm{~m})$ são do tipo illita hexagonal não-filamentosa e do politipp $2 \mathrm{M}$. Moore \& Reynolds (1989) descrevem uma seqüência mineralógica da evolução dos minerais illíticos em função da intensidade da diagênese. Segundo os mesmos, corresponde à passagem da diagênese para o começo do metamorfismo, fácies do tipo zeolita, que atinge o tipo muscovita (Miyashiro 1973). Isto ocorre também com as duas amostras intermedi- 
árias $(4.695 \mathrm{~m})$ que contém um mineral do tipo interestratificado illita/paragonita. Todas essas amostras foram datadas entre $138 \mathrm{Ma}$ a $129 \mathrm{Ma}$. Inversamente, as duas frações finas das amostras mais profundas $(\sim 4.910 \mathrm{~m})$ não apresentam uma cristalinidade já tão boa, contém uma proporção significativa de illita $1 \mathrm{M}$ juntamente com clorita. Estas são mais jovens, exibindo idades convencionais de 119 Ma e $102 \mathrm{Ma}$.

Essas observações conduzem a propor um certo tipo de retromorfose. A retromorfose consistiria em uma transformação das illitas 2M, de primeira geração, em illitas $1 \mathrm{M}$, de segunda geração. Esse processo ocorre em condições de temperaturas mais baixas. No momento dessa transformação, as 1llitas $1 \mathrm{M}$ abrem-se em relação ao argônio radiogênico; as illitas $1 \mathrm{M}$ estão situadas a uma profundidade maior que as illitas $2 \mathrm{M}$, onde a temperatura é mais elevada; porém, a temperatura de bloqueio do argônio radiogênico das illitas $1 \mathrm{M}$ é menor. Em decorrência das quatro condições citadas acima, conclui-se que o conteúdo atual do argônio radiogênico das illitas $1 \mathrm{M}$ diminuiu consideravelmente. Conseqüentemente, isso explicaria as idades mais jovens obtidas sobre as idade das illitas $2 \mathrm{M}$.

A presenca de uma pequena quantidade de feldspato potássico poderia também acompanhar essa retromorfose, o que não foi, porém, observado ao microscópio eletrônico de varredura.

Em resumo, as argilas desta sondagem parecem nos indicar a ocorrência de dois episódios diagenéticos, o primeiro, muito quente, ocorrendo entre $140 \mathrm{Ma}$ e $130 \mathrm{Ma}$, e o segundo, mais frio, posterior, ocorrido talvez entre 120 Ma e 100 Ma.

As celadonitas foram extraídas debasaltos alterados. Esse mineral dioctaédrico, sendo rico em potássio, pode ser utilizado para a datação, da mesma forma que a glauconita. Como ele é formado em condições de alteração secundária, portanto neofonnado, pode ser utilizado para datacão deste evento secundário. A neofonnação da celadonita é evidente tanto por suas condicões de cristalização, na interface entre o basalto e os vacúolos preenchidos de quartzo, calcedônia ou ametista, quanto pela sua geoquímica, que prima pela ausência de titânio (Tab. 2). As idades obtidas variam entre $120 \mathrm{Ma}$ e $75 \mathrm{Ma}$, correspondendo ao mesmo intervalo das argilas de filões mineralizados. Todavia, se o diagrama isotópico K/Ar (Fig. 2) fosse confiável, este intervalo poderia ser reduzido a $120 \mathrm{Ma}-109 \mathrm{Ma}$. A interseccão $\left({ }^{40} \mathrm{Ar} /\right.$ $\left.{ }^{36} \mathrm{Ar}\right)_{0}$ que julga-se anômala, e igual a $233 \pm 12$ (la), leva a interpretar, como mais plausível, que se trata de uma reta do tipo mistura de dois componentes, já que as amostras não foram coletadas no mesmo local e os teores de $\mathrm{K}_{2} \mathrm{O}$ são quase todos idênticos. Entretanto, a título de discussão, caberia mencionar os resultados obtidos em argilas diagenéticas extraídas de arenitos da Formacão Furnas em que um episódio de diagênese secundária pôde ser datado entre 120 a 100 $\mathrm{Ma}$. A reta isotópica calculada, no caso, poderia corresponder a um mecanismo de perda do argônio radiogênico. Esta perda deveria ser idêntica para todas as amostras. Se isso ocorreu, a reta no diagrama desloca-se paralelamente a ela mesma e o ponto de intersecção se desloca em direção a valores menores que 295,5, como teria sido no caso das amostras da Formacão Furnas e também nas argilas do Triássico à sudeste da Bacia de Paris, conforme Bonhomme et al. (1992).

Thomaz Filho (1976) datou argilas da Formação Botucatu. Após tratamento com $\mathrm{HC} 1$ diluído, essas argilas apresentaram como resultados idades de mais ou menos $140 \mathrm{Ma}$. Clauer (1981) mostrou que este tratamento define uma idade que pode ser considerada como a de um evento diagenético. Conseqüentemente, este resultado reforça o deste trabalho, que demonstra ter ocorrido um episódio diagenético importante que afetou os sedimentos da Bacia do Paraná em torno de 140 a $130 \mathrm{Ma}$.
As duas apofilitas datadas forneceram resultados semelhantes de $116 \mathrm{Ma}$ e $110 \mathrm{Ma}$, que as capacita também para datar episódios de alteração tardia de derrames vulcânicos.

Comparação dos resultados com o contexto geodinâmico Todas as datações significativas discutidas no parágrafo precedente parecem revelar a existência de três episódios principais:

- Uma diagênese de alta temperatura ocorrida entre 140 e $130 \mathrm{Ma}$;

- Uma diagênese de baixa temperatura ocorrida entre 110 e $100 \mathrm{Ma}$

- A ocorrência de filões mineralizados entre 90 a 67 Ma.

- Estas datações podem ser comparadas às idades dos eventos diagenéticos e às das erupções magmáticas na mesma região.

- A datacão dos eventos diagenéticos foi obtida em duas zonas: na Bacia Potiguar, ao longo da costa norte do Brasil, em que se revelou a existência de uma alteração diagenética das argilas numa formação do Cretáceo Inferior a $79 \mathrm{Ma}$ (Bonhomme et al 1988) e, na Bacia do Benue, Nigéria, em que um metamorfismo de baixo grau afetando a região de Abakaliki foi também datado de 80 Ma (Benkhelil 1987).

- Os derrames basálticos da Bacia do Paraná foram datados pelo método $\mathrm{K} / \mathrm{Ar}(155$ a $100 \mathrm{Ma})$, pelo método $\mathrm{Rb} / \mathrm{Sr}(135,5$ $\pm 3,2 \mathrm{Ma}$ ) (Rocha Campos et al 1989); pelo método ${ }^{39} \mathrm{Ar} /$ ${ }^{40} \mathrm{Ar}$ (135 a $130 \mathrm{Ma}$ ) (Baksi et al 1991) e também pelo método "Ar ${ }^{40} \mathrm{Ar}$ (133 $\pm 1 \mathrm{Ma}$ ) (Renne et al 1992).

- As intrusões alcalinas do Brasil já datadas também pelos métodos $\mathrm{K} / \mathrm{Ar}$ e $\mathrm{Rb} / \mathrm{Sr}$ mostram idades que variam de 136 Ma a 63 Ma (Gomes et al 1990), com duas modas aparentes em torno de $130 \mathrm{Ma}$ e $80 \mathrm{Ma}$.

As rochas alcalinas e alcalinas-ultrabásicas da África Meridional datam principalmente de $130 \mathrm{Ma}$, pelo método $\mathrm{Rb} / \mathrm{Sr}$ (Bristow 1985, Milner et al 1992).

As datações atualmente conhecidas mostram, pois, que as rochas eruptivas dos dois lados do Oceano Atlântico Sul ocorreram durante o mesmo intervalo de tempo que as formações estudadas neste trabalho, ou seja: as mineralizações filonianas $\mathrm{e}$ os eventos diagenéticos que afetaram a seqüểncia sedimentar e vulcânica da Bacia do Paraná. Da mesma forma que para as rochas vulcânicas estes eventos ocorreram entre 135 Ma e $80 \mathrm{Ma}$, sendo que a diagênese de baixa temperatura, estimada em torno de $110 \pm 10 \mathrm{Ma}$, teria afetado apenas a Bacia do Paraná.

A comparação desses resultados com a história geodinâmica do Atlântico Sul mostra que os dois principais episódios colocados em evidência pela radiocronologia estão relacionados a eventos da abertura oceânica. A idade de cerca de $130 \mathrm{Ma}$, atribuída à principal erupção dos basaltos, é também conhecida como a idade do rifting, embora este início não esteja bem definido estratigraficamente (Asmus \& Guazelli 1981, Macedo 1989, Aquino \& Lana 1990). Todavia, os dados radiocronológicos disponíveis e discutidos aqui conduzem a uma outra idade que estaria entre 139 e 132 Ma.

$A$ idade de mais ou menos 80 Ma corresponde à abertura plena do Oceano Atlântico Sul, quando ele se transformou em um verdadeiro oceano. Entende-se como um verdadeiro oceano, aquele em que basculamentos dos blocos cessaram, onde a dorsal oceânica começa a funcionar sem cobertura crustal (sedimentar e magmática) e sob uma grande volume d'agua em que os radiolaritos começam a depositar diretamente sobre o fundo oceânico (Boillot 1990). Todavia, Sibuet \& Mascle (1978) mostram que a isóbata $4 \mathrm{~km}$, que pode ser considerada como representante do verdadeiro fundo oceânico, esta situada atualmente entre as anomalias magnéticas $\mathrm{MO}$ e A34, datadas respectivamente entre $110 \mathrm{Ma}$ e $79 \mathrm{Ma}$. Essa indetermina cão e comparável ao intervalo de tempo determinado pelas datações deste trabalho, que estão compreendidas entre 110 a $67 \mathrm{Ma}$. E neste intervalo que o segun- 
do episódio de intrusões de rochas alcalinas ocorre (Gomes et al. 1990). Sobre o plano da tectônica, a sedimentação uniforme e pelágica, discordante sobre todas as formações precedentes, começa no Aptiano até o limite Albiano-Cenomaniano, conforme a região; isto é, entre $110 \mathrm{Ma}$ e $95 \mathrm{Ma}$ (Haq \& Van Eysinga 1987). Este último intervalo coincide exatamente ao período definido neste trabalho como de diagênese de baixa temperatura.

\section{CONCLUSÕES}

1. Argilas ligadas às mineralizações de fluorita da Mina Santa Catarina (SC) e de urânio em Poços de Caldas (MG) forneceram idades de K/Ar de 87 e 67,5 Ma, respectivamente.

2. Argilas de diagênese da Bacia do Paraná permitiram precisar a existência de um episódio diagenético de alta tempe- ratura entre 140 Ma e 130 Ma e um episódio diagenético de baixa temperatura entre 120 Ma e $100 \mathrm{Ma}$.

3. Datações K/Ar efetuadas em celadonitas e apofilitas de alteração diagenética, nos basaltos da Formação Serra Geral, coloca este evento entre $120 \mathrm{Ma}$ e $110 \mathrm{Ma}$.

4. Todos os dados acima podem ser interpretados à luz dos fenômenos magmáticos decorrentes da abertura do Oceano Atlântico Sul.

5. A ligação no espaço e no tempo entre diagênese e mineralizações filonianas, de um lado, e tectônica extensional, do outro, já foi observada por diversos autores ao longo do Atlântico Norte. Os resultados obtidos neste trabalho foram comparados com os resultados que permitiram estabelecer o modelo do Atlântico Norte. Estes dados apresentam mecanismos idênticos, ocorridos durante a abertura do Attântico Sul.

\section{REFERÊNCIAS BIBLIOGRÁFICAS}

AQUINO, G.S. \& LANA, M.D. 1990. Exploração na Bacia de Sergipe, Alagoas. O "Estado da Arte". Bol. Geoc. Petrobrás, 4(1):75-84.

ASMUS, H.E. \& GUAZELLI, W. 1981. Descrição sumária das estruturas da margem continental brasileira e das áreas oceânicas e continentais, adjacentes. Hipóteses sobre o tectonismo causador e implicações para os prognósticos do potencial de recursos minerais. In: ASMUS, H.E. ed. Projeto Remac. Rio de Janeiro, Petrobrás, CENPES. p. 187-269.

BAKSI, A.K.; FODOR, R.V.; FARRAR, E. 1991. Preliminary results of ${ }^{\mathrm{w}} \mathrm{Ar} /$ ${ }^{19}$ Ar dating studies on rocks from the Serra Geral Flood-Basalt province and Brazilian continental margin. EOS, 72(17):300.

BASTOS NETO, A.C. 1990. Le District à Fluorine de Santa Catarina (Brésil): Mineralisations et Alterations Hydrothermales dans leur Cadre Géodynamique. France. 366 p. (These doct, Université Orleans).

BASTOS NETO, A.C.; CHARVET, J.; TOURAY, J.C.; DARDENNE, M. 1991. Evolution tectonique du district à fluorine de Santa Catarina (Brésil) en relation avec Fouventure de 1'Atlantique Sud. Bull. Soe. Géol. Fr. 162(3):503-513.

BASTOS, A.C.; TOURAY, J.C.; CHARVET, J : DARDENNE, M. 1991. Tectonic setting of vein deposits in the Santa Catarina fluorite disctrict (S. Brazil). In: PAGEL M. \& LEROY, J.L. Source, Transport and Deposition of Metals, eds. Rotterdam, AA. Balkema, Brookfield. p. 423-425.

BENKHELIL, J. I987. Cretaceous deformation, magmatism and metamorphism in the Lower Benue Trough, Nigeria. J. Geol, 22:467493.

BOILLOT, G. 1990. Géologie des Marges Continentales. Paris, Masson. $135 \mathrm{p}$.

BONHOMME, M.G. 1982. Age triassique et jurassique des argues associees aux mineralisations filoniennes et de phénomènes diagénétiques tardifs en Europe de 1 'Quest. Context géodynamique et implications génétiques. C.R. Acad. Sei., Ser. D, 294:521-524.

BONHOMME, M.G. 1987. Type of sampling and comparison between K-Ar and $\mathrm{Rb}-\mathrm{Sr}$ isotopic dating of fine fractions from sediments in attempt to date young diagenetic events. Chem. Geol.. 65:209-222.

BONHOMME, M.G.; APRAHAMAN, J.; CABY, R.; MISUSAKI, M.P.; THOMAZ, A., P 1988. Three examples of radiogenic argon excess in diagenetic, anchimetamorphic and high-pressure metamorphic environments. In: INTERN. CONE GEOCHEM. EVOLUTION OF THE CONTINENTAL CRUST. Poços de Caldas, 1988. Proceedings... Poços de Caldas, USP. p. 175-195

BONHOMME, M.G.; BAUBRON, J.C.; JEBRAK, M. 1987. Mineralogie, géochimie, terres rares et age $\mathrm{K} / \mathrm{Ar}$ des argues associees aux mineralisations filoniennes. Chem Geol 65:321-339.

BONHOMME, M.G., BÜHMANN, D.; BESNUS, Y. 1983. Reliability of clays and silicifications associated with vein mineralizations in Western Europe. Geol Rundsch., 72(1): 105-117.

BONHOMME, M.G.; MEUNIER, A.; BEAUFORT, D. 1992. K/Ar systematic of clays fractions from Triassic and Liassic sandstones and from small fissures in the basement of the Sancerre-Couy core-drill. In: VIE SYMP. INTERN. OBSERV. CONTINENTAL. CRUST THROUGH DRILLING. Paris, 1992. Proceedings... Paris, Entidade, p. 7-10.

BRISTOW, J.W. 1985. (ed) Alkaline and alkaline-ultrabasic rocks and their xenoliths. Geol. Soc. S. Afr. Trans., 88(2).(Spec. Vol.).

CLAUER.N. 1981. Rb-Sr and K-Ar dating of Precambrian clays and glauconies. In: TROMPETTE, R. \& YOUNG, G. (eds). Upper Precambrian Correlations. Precambrian Res., 15(Sp/Issue):331-352.

CORDANI, U.G. \& HASUI, Y. 1968. Idades K/Ar das rochas alcalinas do Primeiro Planalto do Estado de São Paulo. In: CONOR. BRAS. GEOL, 22. Anais... Belo Horizonte, SBG. p. 149-154.

DOS SANTOS, R.P. \& BONHOMME, M.G. 1991. K/Ar dating of clays associated with flourite mineralizations along the Atlantic coast of South America - Relationships with South Atlantic Ocean Opening. In: PAGEL, M. \& LEROY, J.L eds. Source, Transport and Deposition of Metals. Rotterdam, Balkema, Brookfield. p. 381-384.

GALLAHAN, W.E. \& DUNCAN, R.A. 1991. K-Ar ages of celadonites from the Troodos ophiolite. Cyprus: an new estimate for the duration of hydrothermal alteration in oceanic crust, EOS, 72(44):453.

GOMES, C.B.; RUBERTI, E.; MORBIDELLI, L 1990. Carbonatite complexes from Brazil, a review. J. South Am. Earth. ScL, 3(1):51-63.

GOVINDARAJU, K. 1987. Compilation report on Ailsa Craig granite AC-E with the participation of 128 CIT-IWG laboratories. Geost. Newslett., 11(2):203-255.

GUIRAUD, R. \& MAURIN, J.C. 1991. Le rifting en Afrique au Crétacé Inférieur: synthèse structural, mise en evidence de deux étapes dans la gênese des bassins, relations avec les ouvertures océaniques périafricaines. Bull. Soe. Géol. France. 162(5):811-823.

HALLIDAY, A.N. \& MITCHELL J-G. 1984. K-Ar ages of concentrates from the mineralization of the Pedvoches batholith, Spain, and evidence for Mesozoic hydrothermal activity associated with the break up of Pangea. Earth Planet. Sci. Lett., 68(2):229-239.

HAQ, B.U. \& VAN EYSINGA, F.W.B. 1987. Geological Time Table. Fourth revised, enlarged and updated edition. Amsterdam, Elseviers. $1 \mathrm{p}$, chart.

JEBRAK, M. 1984 Contributional'HistoireNaturelledesFilons (F,Ba) du Domaine Varisque Francois etMarocain. France. 486 p. (These Doct, Université Orleans).

LIEWIG, N.; CLAUER, N.; SOMMER, F. 1987. Rb-Sr and K-Ar dating of clay diagenesis in Jurassic sandstone oil reservoir. North Sea. Am.Assoc. Petrol. Geol Bull, 71(12):1467-1474.

MACEDO, J.M. 1989. Evolução tectônica da Bacia de Santos e áreas continentais adjacentes. Boi Geoc. Petrobrás, 3(3): 159-174.

MAHOOD, G.A. \& DRAKE, R.E. 1982. K-Ar dating young volcanic rocks, a case study of the Sierra La Primavera, Jalisco, Mexico. Geol. Soc. Am. Bull, 93:1232-1241.

MILNER, S.C.; DUNCAN, A.R.; EWART, A. 1992. Quartz Itite sheoijuinhite flows of the Etendeka Formation, north-western Namibia. Bull VolcanoL. 54(3):200-219.

MIYASHIRO, A 1973. Metamorphism and Metamorphic Belts. London, George Allen \& Unwin. $492 \mathrm{p}$.

MOORE, D.M. \& REYNOLDS, R.C.J. 1989. X-Ray Diffraction and the Identification and Analysis of Clay Minerals. Oxford, Oxford Univ. Press. $332 \mathrm{p}$.

MOSSMANN, J.R. 1987. Conditions Physico-Chimiques de Reservoir Gréseux. Approche Petrologique, Minéralogique et Isotopique. Application aux Grès Rhétiens du Bassin de Paris. Strasbourg. 124 p. (These Doct., Univ. L Pasteur).

MOSSMANN, J.R. 1991. K-Ar dating of authigenic illite-smectite material: application to complex mixtures of mixed-layer assemblages. Clay Min. 26(2):189-198.

RENNE, P.R.; ERNESTO, M.; PACCA, I.O.; NARDY, A.J.R.; COE, R.S.; GLEN, J.M.; PRÉVOT, M.; PERRIN, M. 1992. Age and duration of Paraná flood volcanism in Brazil. EOS, 73(43):531-532.

RESPAUT, J.P.; CATHELINEAU, M.; LANCELOT, J.R. 1990. Multistage evolution of the Pierres-Plantees uranium ore deposit (Margeride, France): evidence from mineralogy and U-Pb systematics. Eur. J. Min., 1 : 85-104.

ROCHA CAMPOS, A.C.; CORDANI, U.G.; KAWASHITA, K.; SONOKI, H.M.; SONOKI, I.K. 1989. Age of the Paraná flood volcanism. In: PICCIRILLO, E.M. \& MELFI, A.J. eds. The Mesozoic Flood Volcanics

of the Paraná Basin. Petrogenetic and Geophysical Aspects. São Pau-

lo, IAG/USP. p. 25-45.

RONCHI, L.H.; TOURAY, J.C.; MICHARD, A.; DARDENNE, M.A. 1993. The Ribeira Fluorite district - Southern! Brazil: geological, and geochemical (REE, Sm-Nd isotopes) characteristics. Mineral. Deposita.. (no prelo).

SIBUET, J.C. \& MASCLE, J. 1978. Plate kinematics: implications of Atlantic equatorial fracture zone trends. J. Geophys. Res., 83(87):3401-3421.

STEIGER, R.H. \& JÃGER, E. 1977. Subcommision on geochronology: convention on the use of decay constants in geo and cosmochronology. Earth Planet. Sci. Lett.. 36:359-362.

THOMAZ FILHO, A 1976. Potencialidades doMétodo Rb-Sr para Datação de Rochas Sedimentares Argilosas. São Paulo. 128 p. (Tese de Doutoramento, IG-USP). 
TOURAY, J.C. \& BELLON, H. 1976. Observations à Ia note: "Sur Ia signification paléogéographique de certaines mineralisations filoniennes à fluorine-barytine" de J. Barbier. Bull. Soe. Géol. de France, 7(Mem. H):93-94.

WILLIAMSON, J.H. 1968. Least-squares fitting of a straight line. CanJ.Phys., 46:1845-1847.

ZALAN, P. V ; CONCEIĈ̃O, J.C.J.; WOLFF, S.; ASTOLFI, M.A.M VIEIRA, I.S.; APPI, V.T.; SANTOS NETO, E. V; CERQUEIRA
J.R.; ZANOTTO, O.A.; PAUMER, M.L. 1986. Análise da Bacia do Paraná, GT-Os-009/85. Rio de Janeiro, Petrobrás/Depex. (Relatório SIEX 103-05765 S, v. 4).

MANUSCRITO A729

Recebido em 14 de julho de 1992 Revisão do autor em 23 de fevereiro de 1994 Revisto aceita em 23 de fevereiro de 1994 